OPEN ACCESS

Edited by:

Xinhua Chen,

Fujian Agriculture and Forestry

University, China

Reviewed by:

Xiangmin Lin

Fujian Agriculture and Forestry

University, China

Minh-Thu Nguyen,

Institute of Medical Microbiology, University Hospital Münster, Germany

${ }^{*}$ Correspondence:

Qingpi Yan

yanqp@jmu.edu.cn

Lili Lin

linl/@hxxy.edu.cn

Specialty section: This article was submitted to Antimicrobials, Resistance and Chemotherapy,

a section of the journal

Frontiers in Microbiology

Received: 05 June 2021

Accepted: 21 July 2021

Published: 16 August 2021

Citation:

Hu L, Zhao L, Zhuang Z, Wang $X$, $F u Q$, Huang $H$, Lin $L$, Huang $L$, Qin $Y$, Zhang J and Yan Q (2021) The Effect

of tonB Gene on the Virulence

of Pseudomonas plecoglossicida and the Immune Response

of Epinephelus coioides.

Front. Microbiol. 12:720967.

doi: 10.3389/fmicb.2021.720967

\section{The Effect of tonB Gene on the Virulence of Pseudomonas plecoglossicida and the Immune Response of Epinephelus coioides}

\author{
Lingfei Hu ${ }^{1}$, Lingmin Zhao' ${ }^{1}$, Zhixia Zhuang ${ }^{2}$, Xiaoru Wang ${ }^{2}$, Qi Fu' ${ }^{2}$, Huabin Huang ${ }^{2}$, \\ Lili Lin ${ }^{2 *}$, Lixing Huang ${ }^{1}$, Yingxue Qin1, Jiaonan Zhang ${ }^{3}$ and Qingpi Yan ${ }^{1,2,3 *}$
}

${ }^{1}$ Fisheries College, Jimei University, Xiamen, China, ${ }^{2}$ College of Environment and Public Health, Xiamen Huaxia University, Xiamen, China, ${ }^{3}$ Key Laboratory of Special Aquatic Feed for Fujian, Fujian Tianma Technology Company Limited, Fuzhou, China

Pseudomonas plecoglossicida is the causative agent of "visceral white spot disease" in cultured fish and has resulted in serious economic losses. tonB gene plays a crucial role in the uptake of nutrients from the outer membranes in Gram-negative bacteria. The previous results of our lab showed that the expression of tonB gene of $P$. plecoglossicida was significantly upregulated in the spleens of infected Epinephelus coioides. To explore the effect of ton $B$ gene on the virulence of $P$. plecoglossicida and the immune response of $E$. coioides, ton $B$ gene of $P$. plecoglossicida was knocked down by RNAi; and the differences between the wild-type strain and the tonB-RNAi strain of $P$. plecoglossicida were investigated. The results showed that all of the four mutants of $P$. plecoglossicida exhibited significant decreases in mRNA of tonB gene, and the best knockdown efficiency was $94.0 \%$; the survival rate of $E$. coioides infected with the tonB-RNAi strain was $20 \%$ higher than of the counterpart infected with the wild strain of $P$. plecoglossicida. Meanwhile, the E. coioides infected with the tonBRNAi strain of $P$. plecoglossicida carried less pathogens in the spleen and less white spots on the surface of the spleen; compared with the wild-type strain, the motility, chemotaxis, adhesion, and biofilm formation of the tonB-RNAi strain were significantly attenuated; the transcriptome data of $E$. coioides infected with the tonB-RNAi strain were different from the counterpart infected with the wild strain of $P$. plecoglossicida; the antigen processing and presentation pathway and the complement and coagulation cascade pathway were the most enriched immune pathways. The results indicated that ton $B$ was a virulence gene of $P$. plecoglossicida; ton $B$ gene was involved in the regulation of motility, chemotaxis, adhesion, and biofilm formation; ton $B$ gene affected the immune response of $E$. coioides to $P$. plecoglossicida infection.

Keywords: Pseudomonas plecoglossicida, tonB, pathogenicity, Epinephelus coioides, immune response 


\section{INTRODUCTION}

Pseudomonas plecoglossicida is the causative agent of "visceral white spot disease" in Epinephelus coioides and Larimichthys crocea under the water temperatures of $15-20^{\circ} \mathrm{C}$ and has resulted in high mortality and heavy economic loss (Zhang et al., 2018; Huang et al., 2020). To alleviate the harm caused by $P$. plecoglossicida, its pathogenic mechanism has attracted much attention. The pathogenicity of pathogens was reported to be controlled by different genes. So far, many genes have been shown to have a strong relationship with the virulence regulation of aquatic pathogens, such as $\operatorname{sod} A$ and $\operatorname{sodB}$ to Aeromonas hydrophila (Zhang et al., 2019); $\sec A$ and cheB to Vibrio alginolyticus (Guo et al., 2018); ssaV to Edwardsiella piscicida (Edrees et al., 2018); toxA and toxB to Vibrio parahaemolyticus (Singhapol and Tinrat, 2020); and secY and tssD-1 to P. plecoglossicida (Luo et al., 2020; Ye et al., 2021). The previous transcriptome data (NCBI, SRP115064) of our lab showed that tonB gene of $P$. plecoglossicida was highly expressed in the spleen of $E$. coioides, which suggested that it might play a role in the virulence of $P$. plecoglossicida.

ton $B$ gene encodes TonB, which is an element of the TonB system (TonB-ExbB-ExbD). The TonB system occupies a crucial position in the transport of nutrients, including iron, carbohydrates, hemin, transition metal elements, and vitamin B12 (Schauer et al., 2008; Huang and Wilks, 2017; GomezSantos et al., 2019). In Gram-negative bacteria, the TonB system and tonB-dependent transporter (TBDT) accomplish jointly transport of nutrients. TBDT can acquire energy to transport nutrients from the outer membrane with the help of the TonB system (Oeemig et al., 2018; Kopp and Postle, 2020; Samantarrai et al., 2020). Dong et al. (2019) found that the pathogenicity of $A$. hydrophila was attenuated due to the deletion of ton $B$ gene. However, there have been no reports about $P$. plecoglossicida ton $B$ gene.

Considering the great harms of $P$. plecoglossicida to aquaculture and the potentially important role of ton $B$ in the pathogenicity of $P$. plecoglossicida, this article is devoted to exploring the contribution of ton $B$ in the virulence of $P$. plecoglossicida and the immune response of E. coioides to $P$. plecoglossicida infection. tonB gene of $P$. plecoglossicida was stably knocked down by RNAi; the spleens at 3 and 5 day post injection (dpi) infected with the wild-type strain or the tonB-RNAi strain were sampled and subjected to RNA-seq to monitor the transcriptomes of E. coioides, and the transcriptome data were compared and analyzed.

\section{MATERIALS AND METHODS}

\section{Bacterial Strains and Culture Conditions}

The virulent wild-type strain of P. plecoglossicida (NZBD9) was isolated from the spleen of large yellow croaker suffered from "visceral white spot disease" and was stored at $-80^{\circ} \mathrm{C}$ (Huang et al., 2019). The tonB-RNAi strain of $P$. plecoglossicida was constructed from the wild-type strain of P. plecoglossicida. P. plecoglossicida was routinely grown in
Luria Bertani (LB) broth under 18 or $28^{\circ} \mathrm{C}$ with shaking at $220 \mathrm{rpm}$. Escherichia coli $\mathrm{DH} 5 \alpha$ was obtained from TransGen Biotech (Beijing, China) and grown in $\mathrm{LB}$ broth at $37^{\circ} \mathrm{C}$ with shaking at $220 \mathrm{rpm}$.

\section{RNAi-Induced Knockdown of Pseudomonas plecoglossicida tonB Gene}

Pseudomonas plecoglossicida tonB gene was knocked down according to the methods described by Choi and Schweizer (2006) and Darsigny et al. (2010), with minor modifications. Four oligonucleotides complementary to short hairpin RNA (shRNA) sequences targeting ton $B$ gene (Supplementary Table 1) were designed by BLOCK-iT ${ }^{\mathrm{TM}} \mathrm{RNAi}$ Designer ${ }^{1}$ and synthesized by Shanghai Generay Biotech Co., Ltd. (Shanghai, China). The oligonucleotides were ligated to pCM130/tac vector using T4 DNA ligase (Takara Biomedical Technology, Beijing, China), according to the manufacturer's recommendations. The recombinant plasmids were transformed into competent E. coli DH5 $\alpha$ cells by heat shock and electroporated into $P$. plecoglossicida NZBD9 as described by Luo et al. (2019). The mRNA level of tonB gene in four mutants was determined by quantitative real-time polymerase chain reaction (qRT-PCR).

\section{Growth Rate Assay}

The bacterial suspension were adjusted to optical density at a wavelength of $600 \mathrm{~nm}\left(\mathrm{OD}_{600}\right)=0.3$ and diluted 1,000-fold with LB broth. Aliquot of $200 \mu \mathrm{l}$ of the bacterial diluent was added into per well of 96 -well plate and incubated at $28^{\circ} \mathrm{C}$. The $\mathrm{OD}_{600}$ values of bacterial culture were measured hourly for $48 \mathrm{~h}$ (Zuo et al., 2019). Ten replicates were carried out for each group.

\section{Bacterial Chemotaxis Assay}

The bacterial chemotaxis assay for $P$. plecoglossicida was performed with fine-tuning as described by Zhang et al. (2020). The overnight culture of $P$. plecoglossicida was adjusted to $\mathrm{OD}_{600} \mathrm{~nm} \approx 1.0$ with sterile phosphate-buffered saline (PBS), and $0.25 \mathrm{ml}$ of bacterial suspension was aspirated into a 1-ml syringe. Then, a capillary tube (inner diameter of $0.1 \mathrm{~mm}$, one end sealed) filled with mucus was dipped into the bacterial suspension and incubated at $28^{\circ} \mathrm{C}$ for $1 \mathrm{~h}$. Finally, the mucus in the capillary was blown out, and the colonyforming unit ( $\mathrm{cfu}$ ) number of $P$. plecoglossicida in the mucus was determined by dilution method of plate counting. Three replicates were performed.

\section{Biofilm Formation Assay}

Biofilm formation assay of $P$. plecoglossicida was performed according to the method described by Mao et al. (2020), with some modifications. P. plecoglossicida at exponential growth period was adjusted to $\mathrm{OD}_{600}=0.2$ by fresh $\mathrm{LB}$ broth. Then, $100 \mu \mathrm{l}$ of diluted bacterial suspension was added into per

\footnotetext{
${ }^{1}$ http://rnaidesigner.thermofisher.com/rnaiexpress/setOption.do?designOption= shrna\&pid $=708587103220684543$
} 
well of microtiter plate and incubated at $28^{\circ} \mathrm{C}$ for $24 \mathrm{~h}$. After that, each well was washed twice with sterile PBS, dyed with $175 \mu \mathrm{l}$ of crystal violet $(0.1 \%)$ for $15 \mathrm{~min}$, washed twice with sterile PBS, and air-dried. Finally, the stained biofilm was solubilized into $200 \mu \mathrm{l}$ of $33 \%$ acetic acid, and the OD values of each well were measured at $590 \mathrm{~nm}$. Eight independent replicates were performed.

\section{Motility Assay}

The soft agar plate motility assay for $P$. plecoglossicida was performed as described by Zhang et al. (2019), with minor modifications. The overnight culture in LB was diluted with PBS and adjusted to $\mathrm{OD}_{600}=0.3$. Bacterial suspension of $1 \mu \mathrm{l}$ was inoculated on LB semisolid agar plates supplemented with $0.4 \%$ agar and incubated at $28^{\circ} \mathrm{C}$ for $16-20 \mathrm{~h}$. The diameters of bacterial colonies were measured. Biological replicates were carried out in triplicate for each group.

\section{Adhesion Assay}

The bacterial adhesion assay was conducted according to the method depicted by Guo et al. (2018), with some modifications. Aliquot of $20 \mu \mathrm{l}$ of sterile mucus was spread uniformly over the $22 \times 22 \mathrm{~mm}$ glass slide area. After the mucus was airdried, $4 \%$ methanol was used to fix mucus for $30 \mathrm{~min}$. Bacterial suspension of $200 \mu \mathrm{l}\left(\mathrm{OD}_{600}=0.3\right)$ was spread equably on the region of mucus on glass slides. Sterile PBS of $200 \mu \mathrm{l}$ instead of bacterial suspension was used as the negative control. After incubation at $28^{\circ} \mathrm{C}$ for $2 \mathrm{~h}$ in a damp chamber, the slides were washed three times with PBS to remove the un-adhered bacterial cells. The adhering bacterial cells on the slide were fixed by $200 \mu \mathrm{l}$ of $4 \%$ methanol for $30 \mathrm{~min}$ and dyed with $0.1 \%$ crystal violet for $3 \mathrm{~min}$. After the unstained crystal violet were washed with PBS, the adhering bacterial cells in 10 randomly selected fields were counted under a microscope $(\times 1,000)$. Five replicates were performed.

\section{Epinephelus coioides Infection and Sampling}

All E. coioides infection experiments were executed completely following the proposals in the "Guide for the Care and Use of Laboratory Animals" set by the National Research Council (Copyright 1996 by the National Academy of Sciences). The animal protocols were officially ratified by the Animal Ethics Committee of Jimei University (Acceptance No. JMULAC201159).

Healthy weight-matched E. coioides were obtained from Zhangzhou (Fujian, China) and were acclimatized at $18 \pm 1^{\circ} \mathrm{C}$ for 10 days in recirculating aquaculture systems.

For survival assay, size-matched E. coioides ( 60 fish per group) were intraperitoneally injected with the wild-type strain or tonBRNAi strain of $P$. plecoglossicida at a dose of $5 \times 10^{4} \mathrm{cfu} / \mathrm{fish}$. In addition, 60 E. coioides intraperitoneally injected with PBS were used as the negative control. The daily mortality of experimental fish was observed and recorded until $10 \mathrm{dpi}$.

For RNA-seq, six spleens of three different groups (wild-type strain group, tonB-RNAi strain group, and PBS group) were sampled at 3 and $5 \mathrm{dpi}$, and two spleens were mixed into one sample. All of the samples were sent to Shanghai Majorbio Biopharm Technology Co., Ltd. (Shanghai, China) for sequencing.

For the pathogen load assay and tonB expression assay, six spleens of E. coioides intraperitoneally infected with the wild-type strain or tonB-RNAi strain of $P$. plecoglossicida were randomly sampled at 1, 2, 3, 4, 5, and 6 dpi. P. plecoglossicida cultured at $18^{\circ} \mathrm{C}$ in vitro was considered as the control.

\section{Quantitative Real-Time Polymerase Chain Reaction}

Quantitative real-time polymerase chain reaction was performed by a QuantStudio 6 Flex real-time PCR system (Life Technologies, Carlsbad, CA, United States). Primers are synthesized by Xiamen Borui Biotechnology, and primer sequences are provided in Supplementary Table 2. The 16S rDNA (Sun et al., 2019) was applied to normalize tonB gene
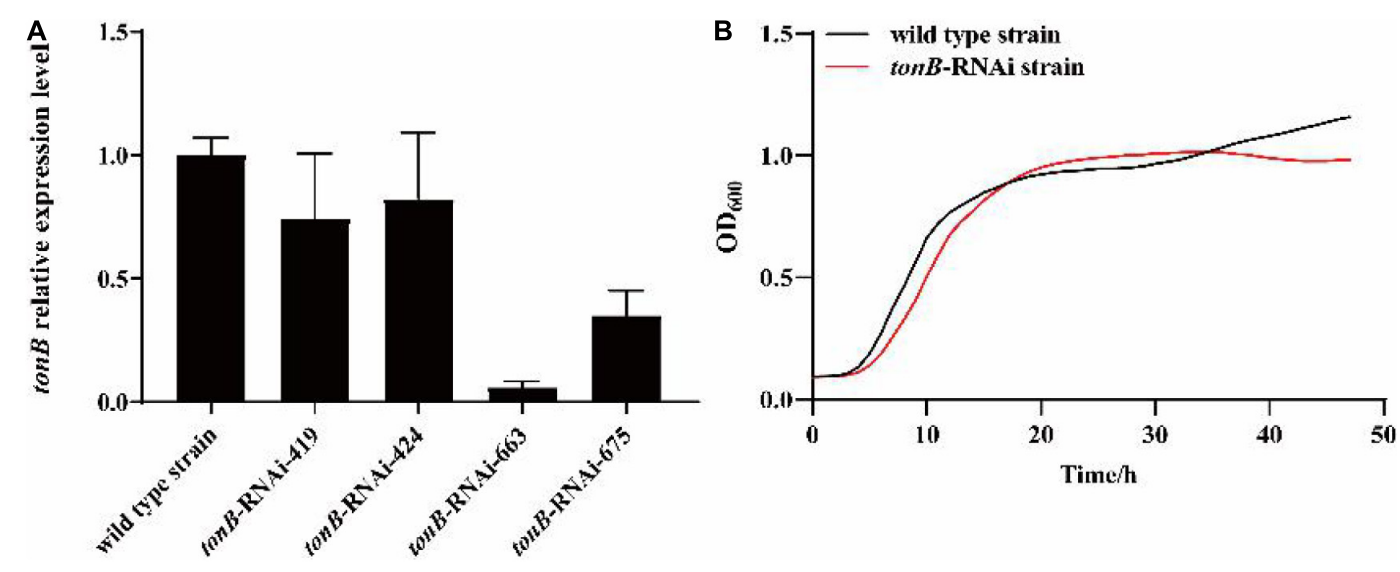

FIGURE 1 | Construction and growth curve of the tonB-RNAi strain of Pseudomonas plecoglossicida. (A) The tonB mRNA levels of four mutant strains. (B) Growth curve of the wild-type strain and tonB-RNAi strain. 
expression levels of $P$. plecoglossicida. The pathogen load of $P$. plecoglossicida in the infected spleens was assessed by the copy number of housekeeping gene gyrB (Xin et al., 2020). The relative expression of gene in different groups was calculated using $2^{-\Delta \Delta C T}$ method (Luo et al., 2020).

\section{Transcriptomic Analysis}

A TruSeq $^{\text {TM }}$ RNA sample preparation Kit (Illumina, San Diego, CA, United States) was used to prepare the RNA-seq libraries under protocols of the Kit. The RNA quality was detected and quantified by the Agilent 2100 Bioanalyzer system (Agilent Technologies, Santa Clara, CA, United States) and the ND-2000 instrument (NanoDrop Technologies, Thermo Fisher Scientific, Waltham, MA, United States) separately. The high-quality RNA samples [OD260/280 $=2.03-2.09$, RNA integrity number $(\mathrm{RIN}) \geq 9.0,28 \mathrm{~S}: 18 \mathrm{~S} \geq 1.0, \geq 1.6 \mu \mathrm{g})$ met the construction of individual sequencing libraries. The rRNA-depleted RNA samples were fragmented in fragmentation buffer, and cDNA synthesis was carried out with protocols supplied with the SuperScript double-stranded cDNA synthesis kit (Invitrogen, Carlsbad, CA, United States). The cDNA libraries were amplified by Phusion DNA polymerase (NEB) after end repair, phosphorylation, and poly(A) addition. Sequencing was performed on the Illumina HiSeq4000 sequencing platform at Majorbio Biotech Co., Ltd. (Shanghai, China). The trimming and quality control of the raw Illumina reads were performed using SeqPrep ${ }^{2}$ and Sickle ${ }^{3}$ with the default settings. The mapped

${ }^{2}$ https://github.com/jstjohn/SeqPrep

${ }^{3}$ https://github.com/najoshi/sickle
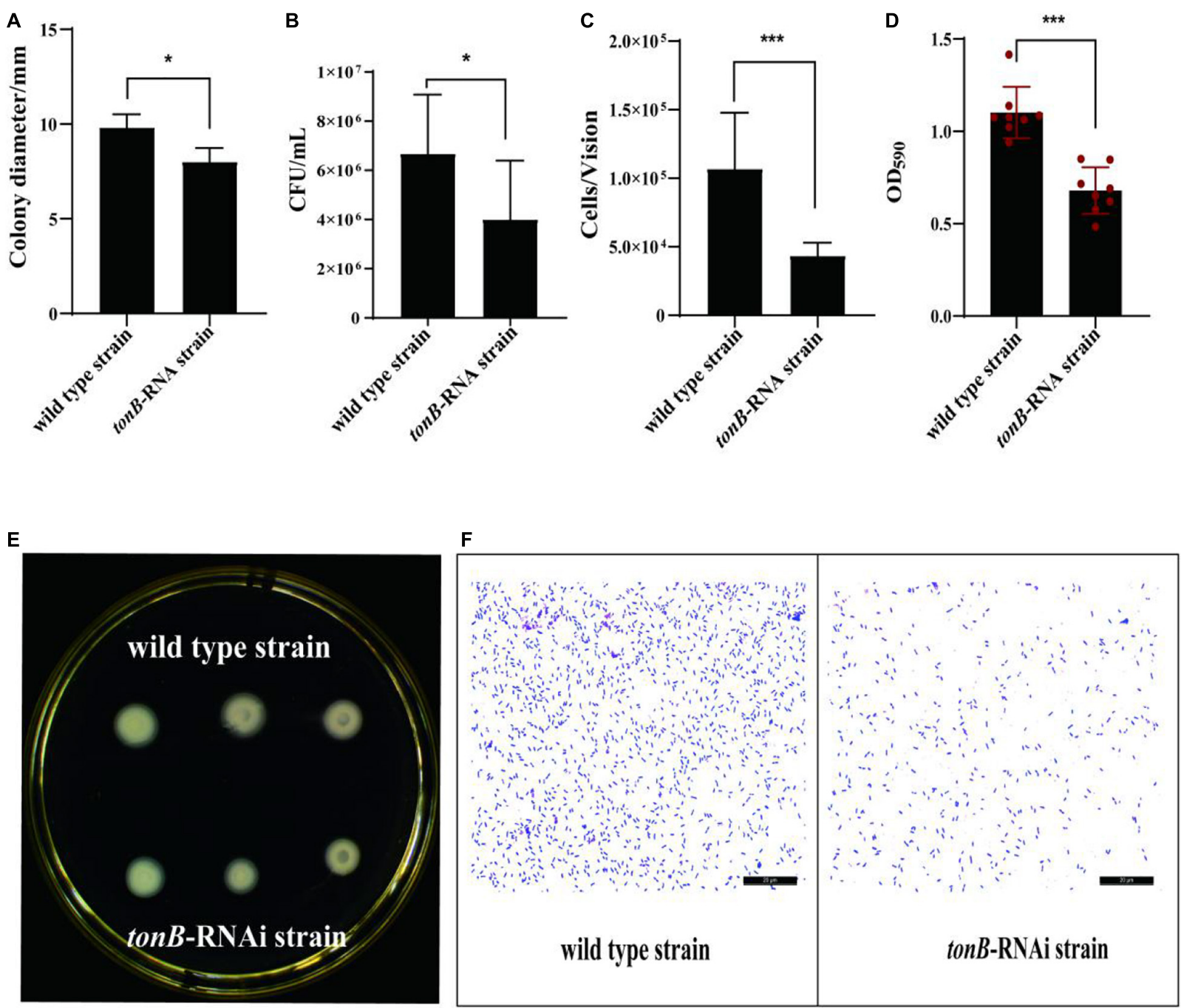

FIGURE 2 | The characteristics of two strains of Pseudomonas plecoglossicida. (A) Colony diameters of wild-type strain and tonB-RNAi strain. (B) Chemotaxis capacity of wild-type strain and tonB-RNAi strain. (C) Adhesion ability of wild-type strain and tonB-RNAi strain. (D) Biofilm formation ability of wild-type strain and tonB-RNAi strain. (E) Colonies of wild-type strain and tonB-RNAi strain on a plate. (F) Adhering bacteria under the microscope. Data are presented as mean \pm SD. ${ }^{*} p<0.05,{ }^{* *} p<0.01,{ }^{* \star *} p<0.001$. 


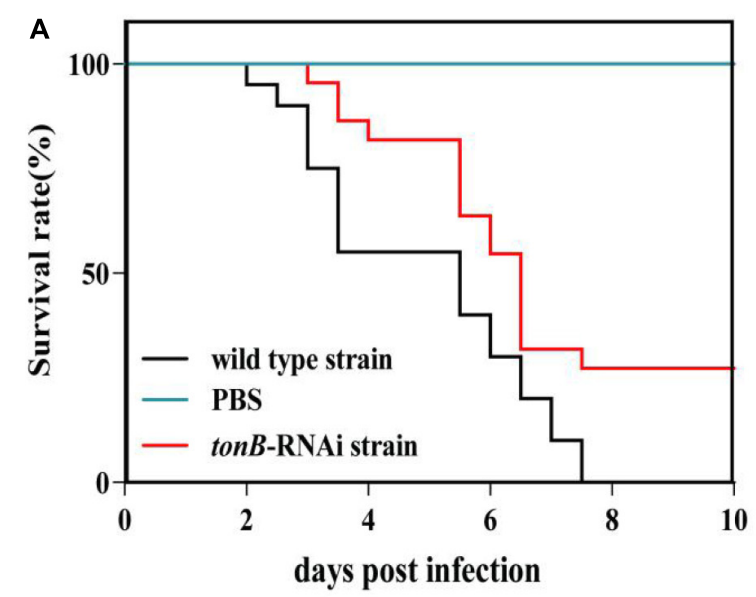

B

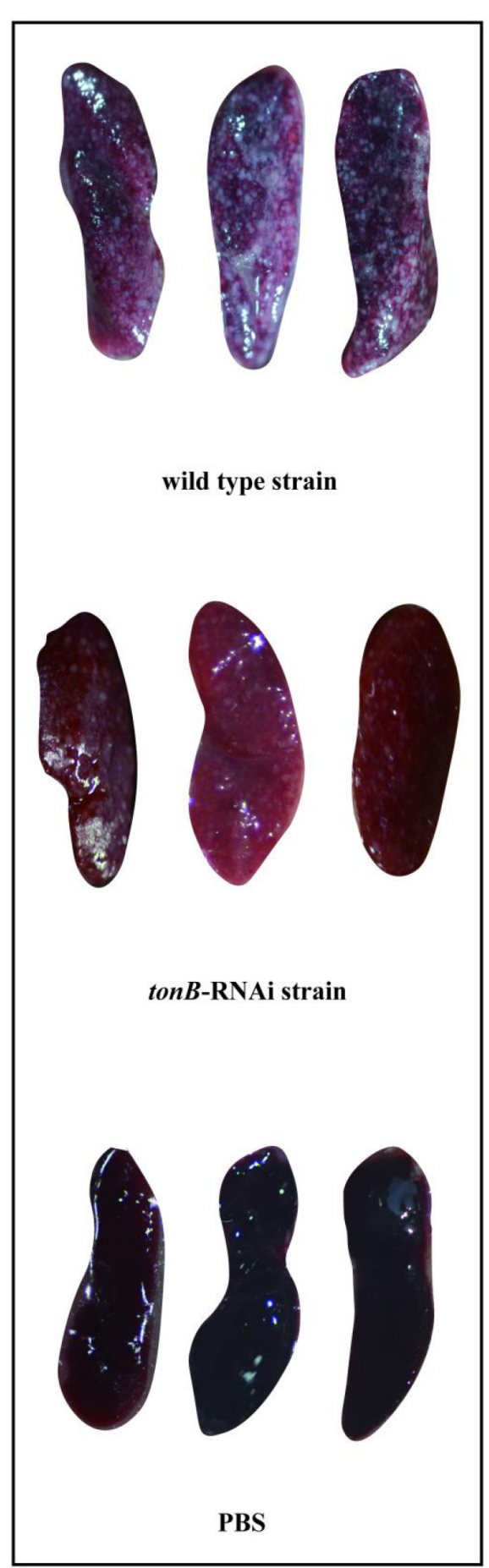

C

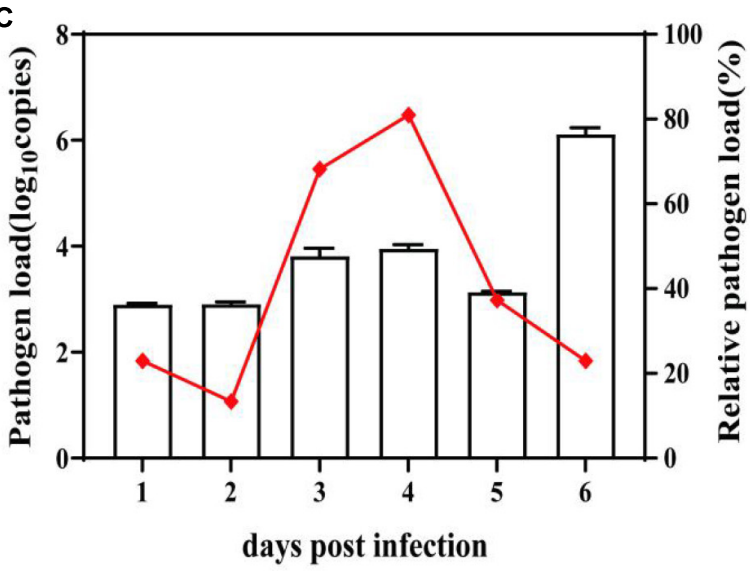

D

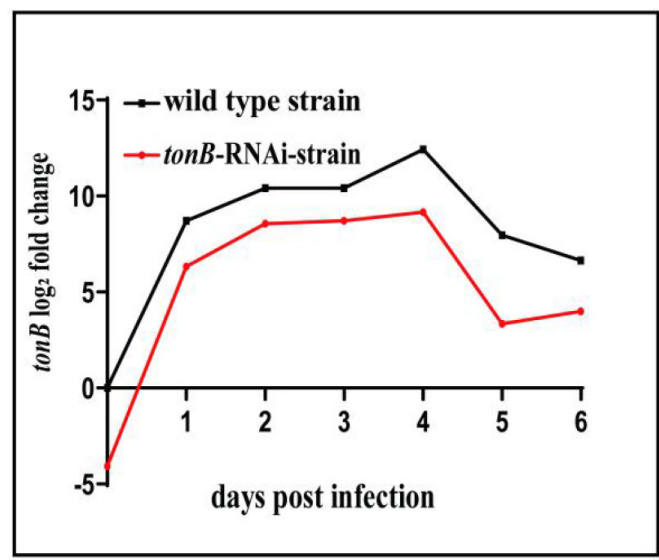

FIGURE 3 | Pathogenicity of two strains of Pseudomonas plecoglossicida to Epinephelus coioides. (A) Survival rate of E. coioides infected with wild-type strain or tonB-RNAi strain. (B) Symptoms of E. coioides spleen after infection of wild-type strain or tonB-RNAi strain. (C) Pathogen load of tonB-RNAi strain of $P$. plecoglossicida in the spleens of $E$. coioides during infection. The bar graph represents the pathogen load and is represented by the copy number of gyr $B$ gene; the line graph represents the relative pathogen load, which is represented by (copy number of gyrB gene of tonB-RNAi strain/copy number of gyrB gene of wild-type strain). (D) mRNA level of tonB gene of P. plecoglossicida in E. coioides spleen during infection. 
reads were used for de novo assembly as the unigenes of E. coioides.

To investigate the biological processes, the BLAST2GO software $^{4}$ was used for the Gene Ontology (GO) annotation (Conesa et al., 2005), which accomplished the molecular annotation of differentially expressed transcripts of E. coioides. The differentially expressed mRNAs (DEMs) met the standards $[|\log 2 \mathrm{FC}| \geq 1$ and false discovery rate $(\mathrm{FDR})<0.05]$ was deemed as significant. The GO enrichment analysis visualization of E. coioides transcriptome data was performed by the clusterProfiler R package (Yu et al., 2012). Finally, metabolic pathways were analyzed with Kyoto Encyclopedia of Genes and Genomes (KEGG) (Okuda et al., 2008). Furthermore, 10 genes were randomly selected from $E$. coioides to verify the reliability of RNA-seq by qRT-PCR (Supplementary Figure 1).

\section{Statistical Analyses}

The experimental data are showed as means \pm SD and dissected with one-way ANOVA followed by Dunnett's test using IBM

${ }^{4}$ https://www.blast2go.com/
SPSS Statistics 26.0 (Armonk, NY, United States). $p<0.05$ was considered as statistically significant.

\section{Data Access}

The RNA sequencing results were put aside in the GenBank SRA database under accession number SRP315640.

\section{RESULTS}

\section{Effect of RNAi on tonB mRNA Level of Pseudomonas plecoglossicida}

After shRNA sequence design and synthesis, recombinant plasmid construction, and electrical transfer, four RNAi mutant strains (tonB-RNAi-419, tonB-RNAi-424, tonB-RNAi-663, and tonB-RNAi-675) were successfully constructed. The results of qRT-PCR showed that tonB gene mRNA levels of four mutants were lower than those of the wild-type strain of $P$. plecoglossicida (Figure 1A). The mutant named tonB-RNAi-663 (hereafter called the ton $B$-RNAi strain) exhibited the best silencing efficiency (94.0\%) and was chosen for further research.

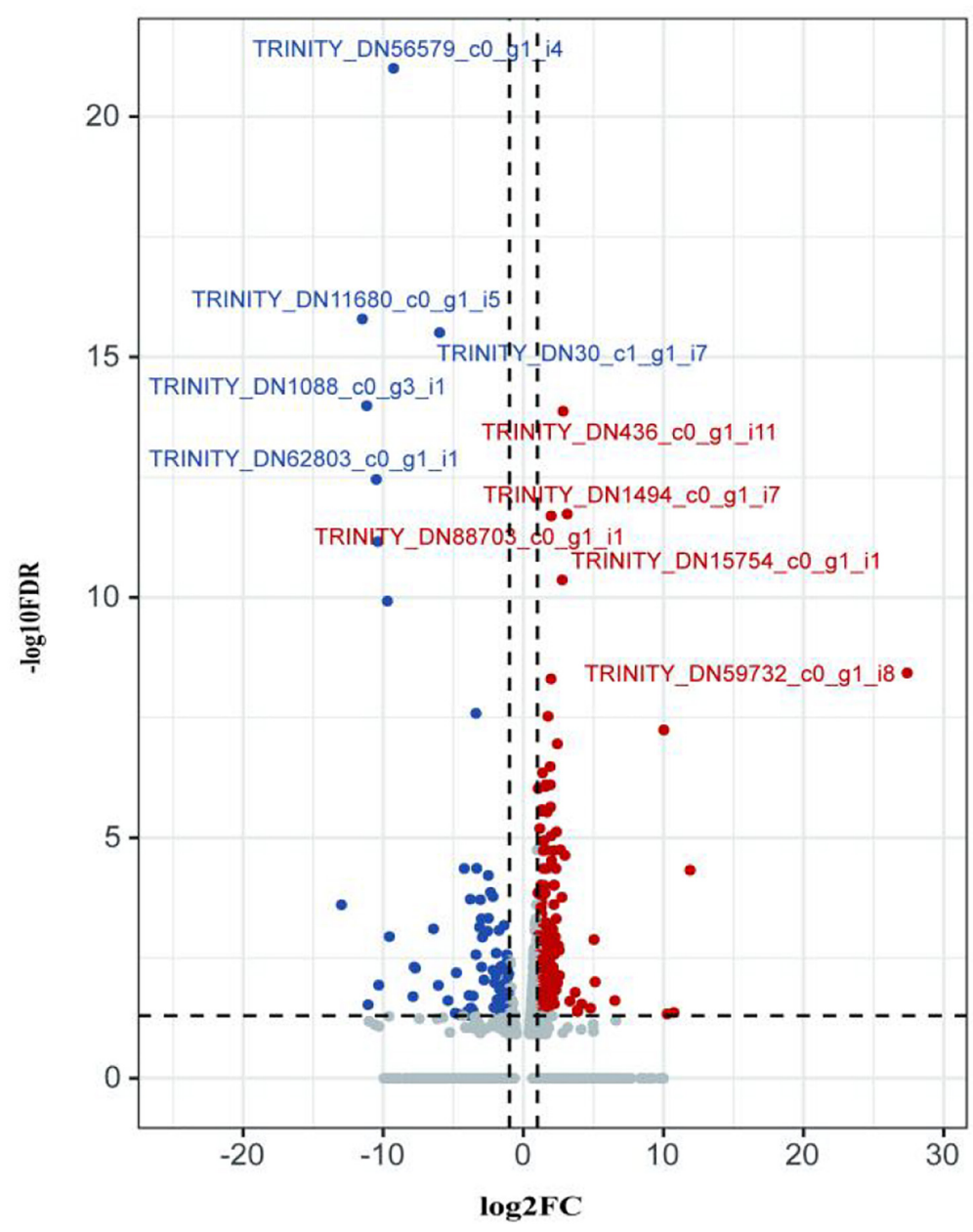

Group

- Down-regulated

- not-significant

- Up-regulated

FIGURE 4 | Volcano plot obtained from the DESeq2 analysis of Epinephelus coioides's spleen RNA pools (3 dpi). 


\section{Effects of tonB Gene Silencing on the Growth of Pseudomonas plecoglossicida}

The growth curve of the tonB-RNAi strain and wild-type strain of $P$. plecoglossicida under the same culture conditions was determined. The results illustrated that there was no significant difference between the growth rate of the tonB-RNAi strain and wild-type strain of $P$. plecoglossicida (Figure 1B).

\section{Effects of tonB Gene Silencing on the Characteristics of Pseudomonas plecoglossicida}

The motility, chemotaxis, and adhesion of the tonB-RNAi strain of $P$. plecoglossicida were enervated compared with the wildtype strain; and the motility, chemotaxis, and adhesion of tonB have weakened by $18.17 \%$ (Figures 2A,E), 39.99\% (Figure
2B), and 59.34\% (Figures 2C,F), respectively, at corresponding periods. Meanwhile, the tonB-RNAi strain of $P$. plecoglossicida showed an extremely significant difference $(p<0.001)$ in biofilm formation as compared with the wild-type strain. When the expression of ton $B$ was inhibited, the biofilm formation ability of P. plecoglossicida decreased at 38.35\% (Figure 2D).

\section{Effects of tonB Gene Silencing on the Virulence of Pseudomonas plecoglossicida}

Infection of the wild-type strain of $P$. plecoglossicida caused the death of E. coioides. The first death was recorded at $2 \mathrm{dpi}$, and the mortality reached $100 \%$ at $7.5 \mathrm{dpi}$. Infection of same dose of the tonB-RNAi strain of $P$. plecoglossicida resulted in 1 day delay in the time of first death and 20\% decrease in

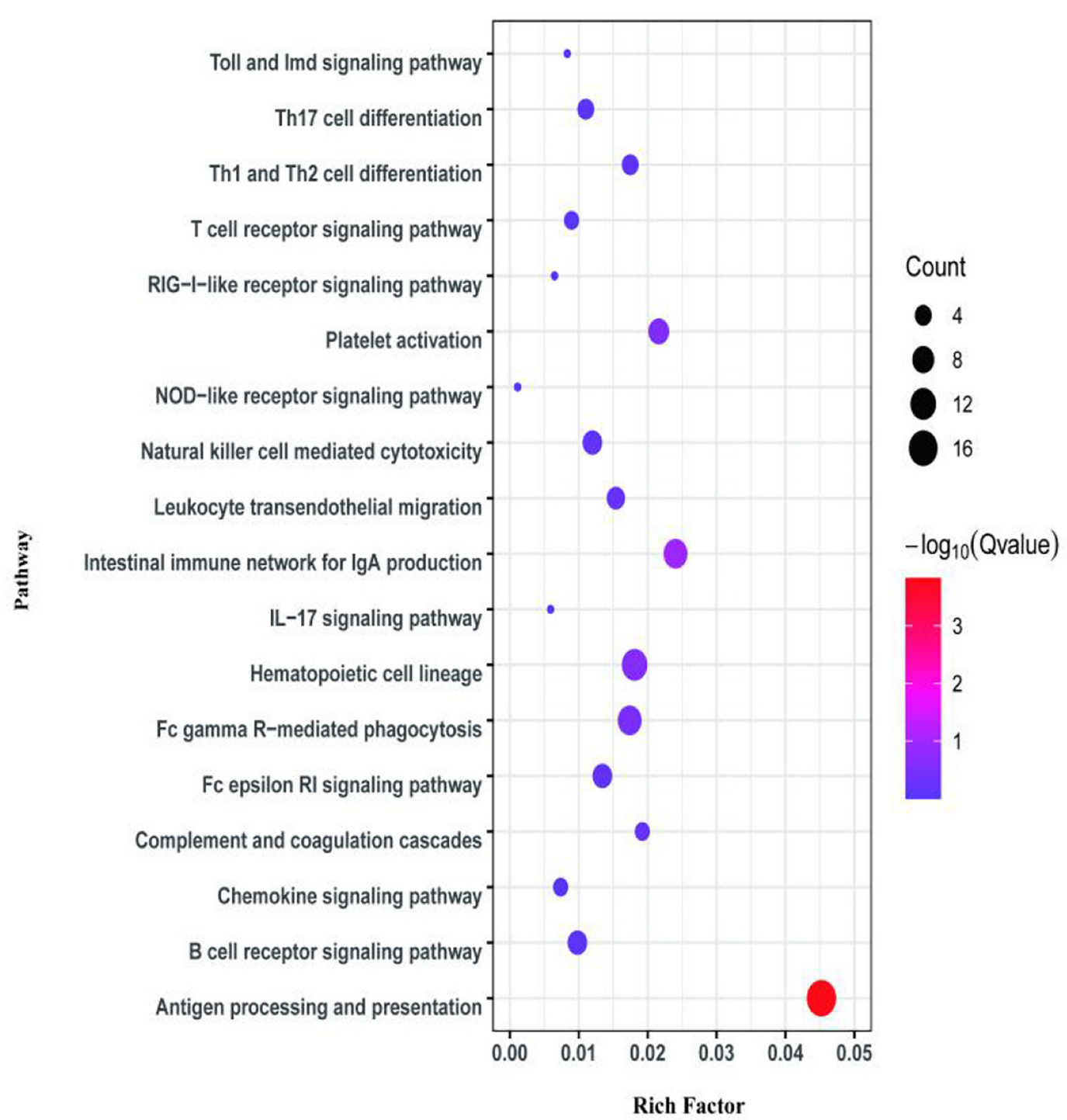

FIGURE 5 | Kyoto Encyclopedia of Genes and Genomes (KEGG) pathway enrichment analysis for differentially expressed mRNAs (DEMs) of transcriptome at 3 dpi. 
cumulative mortality. No death of E. coioides injected with PBS was recorded (Figure 3A).

The surface of E. coioides spleens injected with the wildtype strain of $P$. plecoglossicida was covered with numerous white nodules, while much fewer white nodules were found on the surface of counterpart spleens infected with the tonBRNAi strain, and no white nodule was found on the surface of counterpart spleens injected with PBS (Figure 3B).

The pathogen loads of the tonB-RNAi strain of P. plecoglossicida were always lower than those of the wildtype strain during the whole infection. The relative pathogen load (pathogen load of the tonB-RNAi strain/pathogen load of the wild-type strain) of $P$. plecoglossicida peaked at $4 \mathrm{dpi}$. Although the tonB-RNAi strain had a higher pathogen load at 6 dpi than on other times, the relative pathogen load at 6 dpi was lower than that on other times except that at 2 dpi (Figure 3C). The mRNA level of ton $B$ gene of P. plecoglossicida in the spleen of $E$. coioides was always higher than that in vitro, and the highest values were recorded at $4 \mathrm{dpi}$. Simultaneously, the expression levels of tonB in the tonB-RNAi strain were always lower than those in the wild-type strain (Figure 3D).

\section{The Effects of tonB Gene on the Immune Response of Epinephelus coioides to Pseudomonas plecoglossicida Infection Quality Control of RNA-Seq Data}

The E. coioides's spleens were subjected to RNA-seq after being infected with the tonB-RNAi strain or wild-type strain of P. plecoglossicida. The A/T/G/C base content distribution was

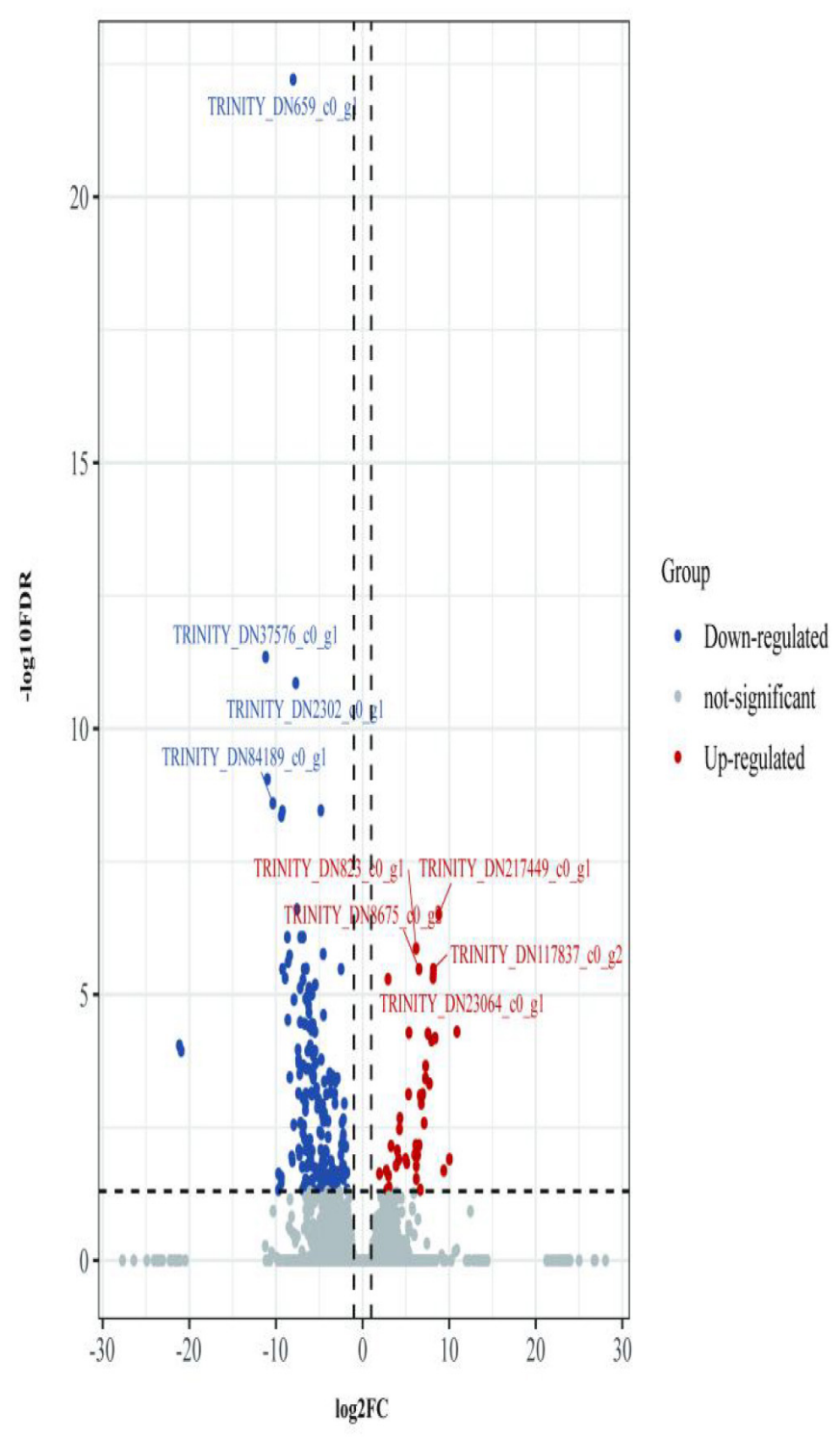

FIGURE 6 | Volcano plot obtained from the DESeq2 analysis of Epinephelus coioides's spleen RNA pools (5 dpi). 
balanced, and N\% met the normative range (Supplementary Figure 2). The main criterion of evaluating the quality of reads was Q20, which fulfilled the requirement of sequencing data of each sample (Q20 > 98\%) (Supplementary Figure 3). The base error rate of the sequencing data was $<0.1 \%$. Pearson's correlation coefficients $(r)$ showed that three biological replicate samples were closely correlated $(r>0.9)$ (Supplementary Figure 4). The quality of sequence data satisfied the requirements for the subsequent data process and analysis steps.

\section{Analysis of Differentially Expressed mRNAs}

DESeq2 was used for the analysis of significantly differentially expressed transcripts of E. coioides. The criteria of the statistically significant of mRNA expression level changes in the transcriptome data were FDR $<0.05$ and $|\log 2 \mathrm{FC}| \geq 1$. GO and KEGG pathway enrichment analyses were performed for DEMs.

The first analyzed transcriptome data from the E. coioides's spleens were sampled at $3 \mathrm{dpi}$. There exist 375 DEMs between the spleen infected with tonB-RNAi strains and wild-type strains of $P$. plecoglossicida, which included 291 upregulated mRNAs and 84 downregulated mRNAs (Figure 4). Further KEGG analysis showed that 20 KEGG pathways involved in immune response were enriched (Figure 5). Of the total DEMs, $26.67 \%$ were enriched in immune system pathways, including Toll and Imd signaling pathway, Intestinal immune network for IgA production, B-cell receptor signaling pathway, and antigen processing and presentation. The antigen processing and presentation pathway (KO ID: ko04612) was significantly enriched based on the $p$-value of Fisher's exact test.

The second analyzed transcriptome data from the E. coioides's spleens were sampled at $5 \mathrm{dpi}$. A total of $218 \mathrm{mRNAs}$ collected from the spleen infected with the tonB-RNAi strain of $P$. plecoglossicida were identified as significant differences in expression compared with the spleen infected with the wild-type strain, which included 43 upregulated mRNAs and 175 downregulated mRNAs (Figure 6). According to the GO annotation conventions, DEMs fall into three categories: biological processes, cellular components, and molecular functions. A total of $117 \mathrm{GO}$ terms were enriched, including 29 significantly enriched GO terms. The 29 notably enriched GO terms of the three categories were selected for statistical analysis. The GO analysis results showed that tonB gene had a great influence on the immune system, because more than half of the immune GO terms were enriched in biological processes (Figure 7). Of the DEMs, 19.3\% were enriched in immune GO terms. According to the KEGG database, 145 KEGG pathways were enriched, including 14 immune-related KEGG pathways. Of the total DEMs, 35.78\% were enriched in immune system pathways (Figure 8). Complement and coagulation cascade pathway (ko04610), which included the greatest number of DEMs, was significantly enriched according to the $p$-value of Fisher's exact test.

The most significant enrichment pathways were complement and coagulation cascades pathway and antigen processing and presentation pathway according to the KEGG enrichment analysis of the transcriptome data that from the E. coioides's

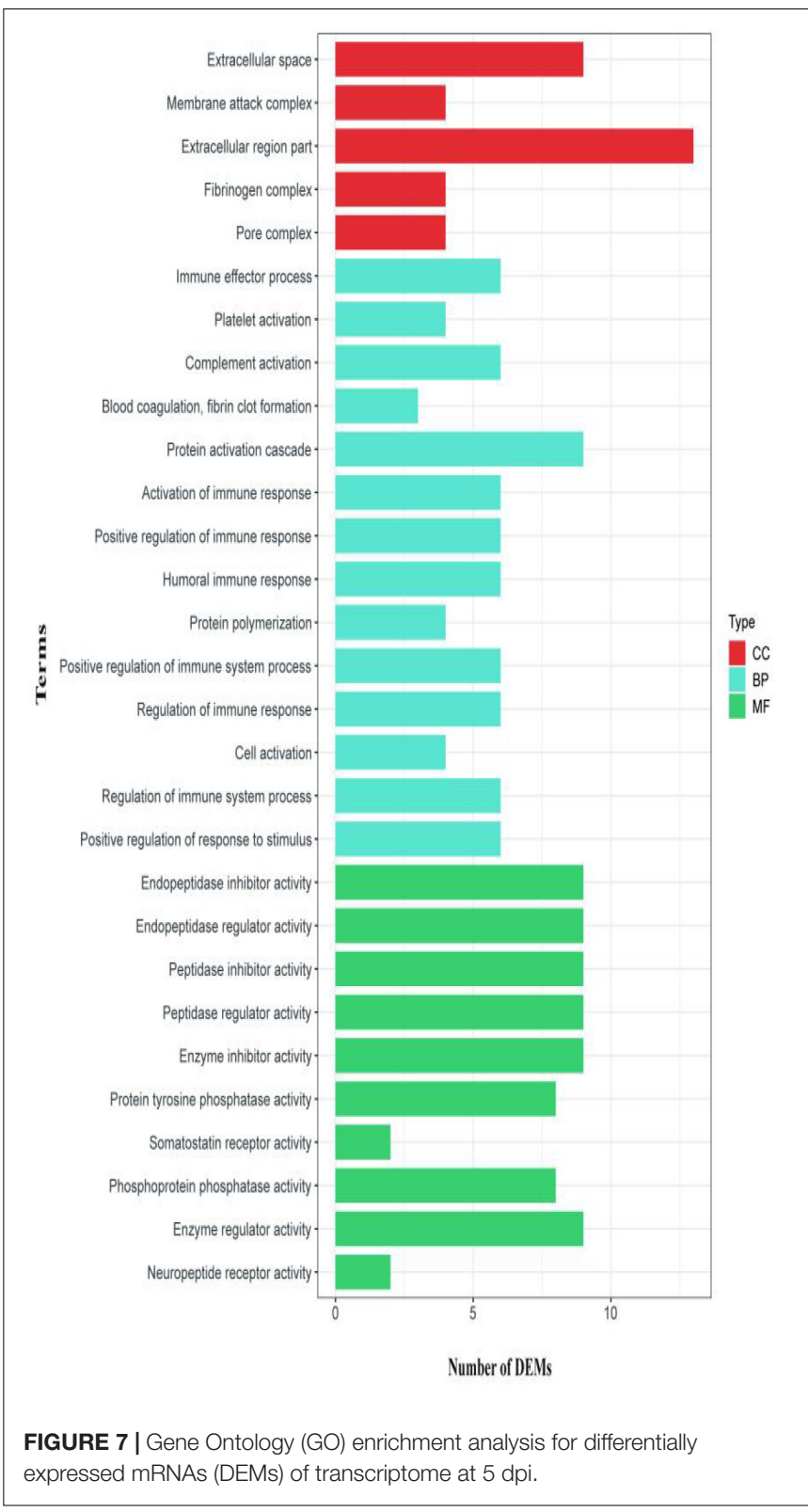

spleens were sampled at 3 and $5 \mathrm{dpi}$, respectively. Antigen processing and presentation pathway enriched 17 DEMs significantly, which including six downregulated DEMs (such as MHCII and TAP1/2) and 11 upregulated DEMs (such as MHCI, AEP, CTSB/L/S, TCR, TAPBP, and HSP70) (Figure 9A). Complement and coagulation cascades pathway enriched 21 downregulated DEMs significantly (such as FI, C3, C5, C6, C7, C8, and C9) (Figure 9B).

\section{DISCUSSION}

RNA interference has been used for many fields to explore the functions of genes (Belles, 2010; Cooper et al., 2021). In this study, four shRNAs exhibited different silence efficiencies to ton $B$ gene, 


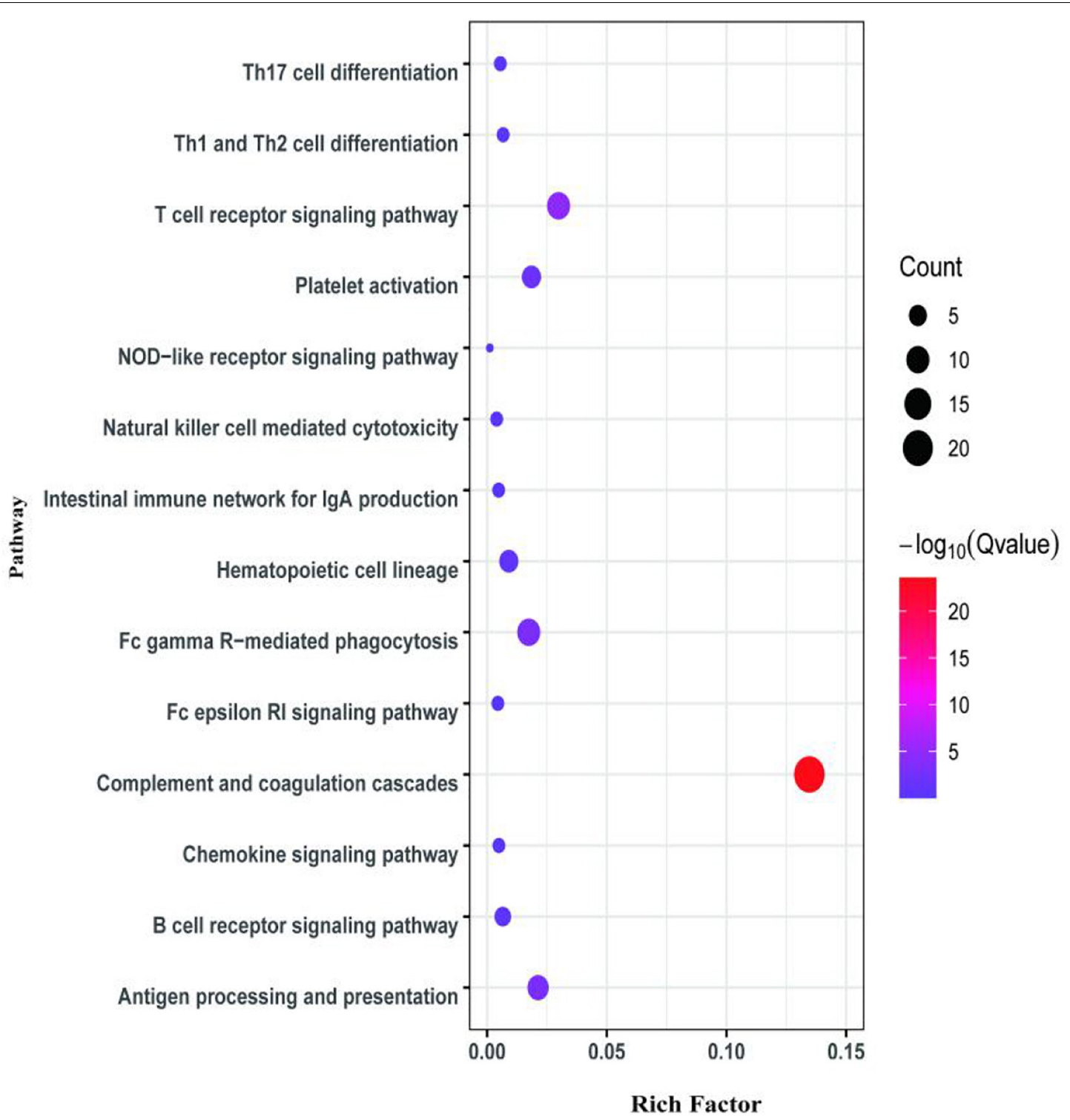

FIGURE 8 | Kyoto Encyclopedia of Genes and Genomes (KEGG) pathway enrichment analysis for differentially expressed mRNAs (DEMs) of the second transcriptome (5 dpi).

which were consistent with the previous RNAi results (Liu et al., 2020; Xin et al., 2020). The best silence efficiency of four shRNAs to $\operatorname{ton} B$ gene was $94 \%$, which was higher than that of $p v d E$ gene (Xin et al., 2020) but lower than that of impB gene (Liu et al., 2020). The stability of gene silencing is crucial to the study of gene function. In the present study, ton $B$ gene in the tonB-RNAi strain of $P$. plecoglossicida was persistently silenced during the infection process, and the relative expressions of ton $B$ gene in the ton $B$ RNAi strain were always lower than those of the wild-type strain. These results indicated that RNAi of tonB gene was reliable and laid the foundation for subsequent research.

The TonB protein, encoded by ton $B$ gene, has amalgamated to form the TonB system with ExbB and ExbD proteins (Liao et al., 2015). Abdelhamed et al. (2017) investigated the virulence of tonB gene of Edwardsiella ictaluri, and their findings show that the E. ictaluri mutant defective in ton $B$ has a 2.16 -fold reduction in virulence as compared with the wild-type of E. ictaluri. Wang et al. (2008) found that the $\mathrm{LD}_{50}$ value of three ton $B$ mutant strains of $V$. alginolyticus was increased by 11-fold, 14-fold, and 25-fold, respectively. RNAi of tonB gene caused a $20 \%$ increase in the survival rate of $E$. coioides to $P$. plecoglossicida infection and had no significant effect on the growth rate of $P$. plecoglossicida, which suggested that the decline in mortality of $E$. coioides was due to the decrease of $P$. plecoglossicida virulence, not due to the decrease of bacterial growth rate. These results suggested that ton $B$ gene contributed to the pathogenicity of $P$. plecoglossicida. These results agreed with previous studies of ton $B$ gene contribution to the virulence 


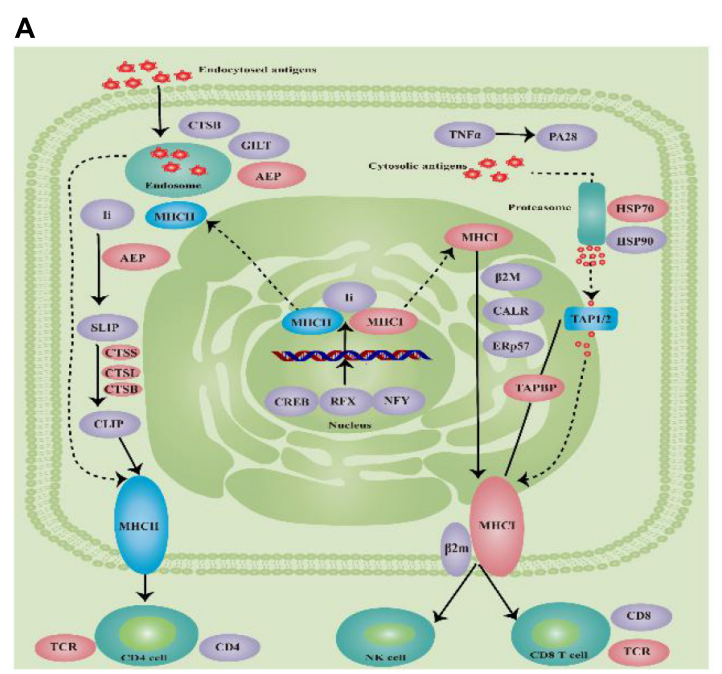

B

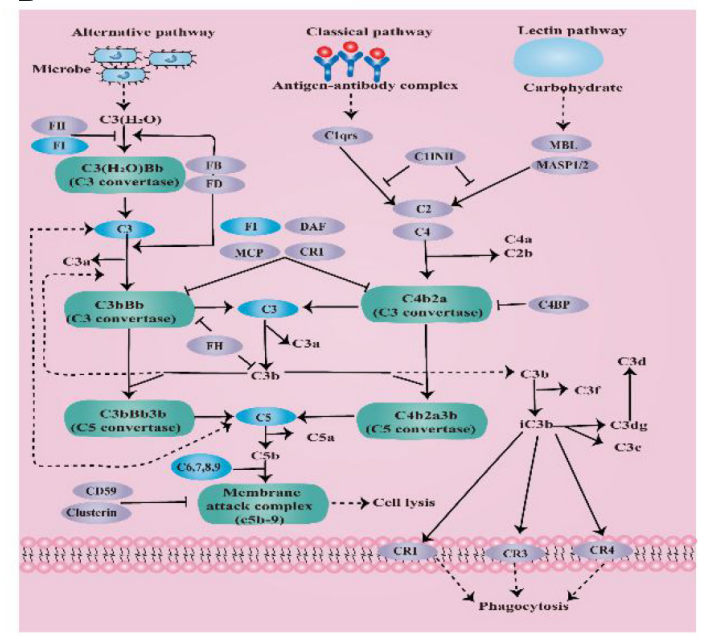

FIGURE 9 | (A) Schematic diagram of antigen processing and presentation pathway. (B) Schematic diagram of complement and coagulation cascades pathway (blue indicates downregulation, red indicates upregulation, and purple indicates no significant change).

in other bacterial strains. The different influence degrees of ton $B$ gene on the pathogenic of different bacteria might be due to the bacterial species, the host species, and the conditions of infection. The results of pathogen load and symptoms agreed with the mortality result. Several genes have been verified to associate with the virulence of P. plecoglossicida (Luo et al., 2019). The silencing of some of these genes in P. plecoglossicida resulted in lower mortality in experimental fish infected with the mutants (Tang et al., 2019; Wang et al., 2019), and silencing of other virulence genes did not result in the death of E. coioides infected with the mutants of P. plecoglossicida (Liu et al., 2020; Xin et al., 2020).

RNAi of tonB gene attenuated bacterial motility, chemotaxis, adhesion, and biofilm formation of P. plecoglossicida. Bacterial motility (Luo et al., 2016), chemotaxis (Zhang et al., 2020), adhesion (Guo et al., 2018), and biofilm formation (Zhang et al., 2019) have been demonstrated to be related to the pathogenic of pathogen. Some studies showed that ton $B$ mutant had great influences on motility, adhesion, and in vivo virulence due to its ability of helping the formation of type IV pili (Huang et al., 2004; Duong-Nu et al., 2016).

Nowadays, transcriptome analysis is an important way to reveal the mechanisms of host immune response to infection (Wu and Chen, 2016; Song et al., 2017; Li et al., 2021). In this study, compared with the transcriptome data from E. coioides infected with the wild strain of P. plecoglossicida, significant changes of transcriptome were observed in the spleen of $E$. coioides infected with the tonB-RNAi strain. The GO analysis results showed that the tonB-RNAi strain had a great influence on the immune system, and more than half of the immune pathways were enriched in biological processes. The KEGG pathway analysis results showed that the most significant pathways for enrichment were complement and coagulation cascades pathway and antigen processing and presentation pathway.
Complement and coagulation cascades pathway is an immune defense mechanism of the host, and the complement cascades are closely associated with coagulation cascades to jointly achieve effective protection of the host (Lupu et al., 2014; Wiegner et al., 2016). It is widely known that the activation and generation of $\mathrm{C} 3 \mathrm{a}$ and $\mathrm{C} 5 \mathrm{a}$ have serious proinflammatory effects (Pontrelli et al., 2020). Compared with the transcriptome data from $E$. coioides infected with the wild-type strain of P. plecoglossicida, all of the DEMs in transcriptome data from E. coioides infected with the tonB-RNAi strain were significantly downregulated in the complement and coagulation cascades pathway, which indicated that the inflammatory reaction significantly declined in E. coioides infected with the tonB-RNAi strain of P. plecoglossicida.

The antigen processing and presentation pathway plays a crucial role in immunological process, which is presented by major histocompatibility complexes (MHCs) (Park and Jae-Hwan, 2011; Wieczorek et al., 2017). MHCI relies on proteasomal proteolysis to present foreign peptides that come from degradation of endocellular microbial pathogens; MHCII depends upon lysosomal degradation to accomplish the presentation and processing of extracellular antigens (Wilson, 2017). Therefore, the determining factor of the successful pathogen elimination obviously depends on the recognition of MHCs in the immune system (Unanue, 2002). Compared with the transcriptome data from E. coioides infected with the wild-type strain of $P$. plecoglossicida, most of the DEMs in transcriptome data from E. coioides infected with the tonB-RNAi strain related with MHCI and MHCII were significantly upregulated in the antigen processing and presentation pathway. These results indicated that E. coioides's immune system could more efficiently identify the tonB-RNAi strain of $P$. plecoglossicida, which might cause the immune system to remove the mutant strain more efficiently. 


\section{CONCLUSION}

In conclusion, ton $B$ is a virulence gene of $P$. plecoglossicida; $\operatorname{ton} B$ gene is involved in the regulation of bacterial motility, chemotaxis, adhesion, and biofilm formation of $P$. plecoglossicida; RNAi of tonB gene significantly affected the immune response of E. coioides to P. plecoglossicida infection; complement and coagulation cascades pathway and antigen processing and presentation pathway are the most affected immune pathways.

\section{DATA AVAILABILITY STATEMENT}

The datasets presented in this study can be found in online repositories. The names of the repository/repositories and accession number(s) can be found in the article/Supplementary Material.

\section{ETHICS STATEMENT}

The animal study was reviewed and approved by the Animal Ethics Committee of Jimei University (Acceptance No. JMULAC201159).

\section{AUTHOR CONTRIBUTIONS}

All authors contributed to the article. QY, LL, ZZ, and XW conceived the experiments. LFH, LL, and LZ conducted

\section{REFERENCES}

Abdelhamed, H., Lawrence, M. L., and Karsi, A. (2017). The role of Edwardsiella ictaluri TonB mutant in Edwardsiella ictaluri virulence. Front. Physiol. 8:1066. doi: 10.3389/fphys.2017.01066

Belles, X. (2010). Beyond Drosophila: RNAi in vivo and functional genomics in insects. Annu. Rev. Entomol. 55, 111-128. doi: 10.1093/bfgp/elp052

Choi, K.-H., and Schweizer, H. P. (2006). mini-Tn7 insertion in bacteria with single attTn7 sites: example Pseudomonas aeruginosa. Nat. Protoc. 1, 153-161. doi: $10.1038 /$ nprot.2006.24

Conesa, A., Gotz, S., Garcia-Gomez, J. M., Terol, J., Talon, M., and Robles, M. (2005). Blast2GO: a universal tool for annotation, visualization and analysis in functional genomics research. Bioinformatics 21, 3674-3676. doi: 10.1093/ bioinformatics/bti610

Cooper, A. M. W., Song, H., Shi, X., Yu, Z., Lorenzen, M., Silver, K., et al. (2021). Characterization, expression patterns, and transcriptional responses of three core RNA interference pathway genes from Ostrinia nubilalis. J. Insect Physiol. 129, 104181-104181. doi: 10.1016/j.jinsphys.2020.10 4181

Darsigny, M., Babeu, J.-P., Seidman, E. G., Gendron, F.-P., Levy, E., Carrier, J., et al. (2010). Hepatocyte nuclear factor- $4 \alpha$ promotes gut neoplasia in mice and protects against the production of reactive oxygen species. Cancer Res. 70, 9423-9433. doi: 10.1158/0008-5472.CAN-10-1697

Dong, Y., Geng, J., Liu, J., Pang, M., Awan, F., Lu, C., et al. (2019). Roles of three TonB systems in the iron utilization and virulence of the Aeromonas hydrophila Chinese epidemic strain NJ-35. Appl. Microbiol. Biotechnol. 103, 4203-4215. doi: 10.1007/s00253-019-09757-4

Duong-Nu, T.-M., Jeong, K., Hong, S. H., Hong-Vu, N., Van-Hoan, N., Min, J.-J., et al. (2016). All three TonB systems are required for Vibrio vulnificus CMCP6 tissue invasiveness by controlling flagellum expression. Infect. Immun. 84, 254-265. doi: 10.1128/iai.00821-15 the experiments. All authors assisted in the collection and interpretation of data. LFH, ZZ, and QY wrote the manuscript.

\section{FUNDING}

This work was supported by the National Natural Science Foundation of China under contract No. 31972836, open fund of Fujian Province Key Laboratory of Special Aquatic Formula Feed under contract No. TMKJZ2002, and the Fund of Fujian Key Laboratory of Functional Aquafeed and Culture Environment Control (FACE20200007).

\section{SUPPLEMENTARY MATERIAL}

The Supplementary Material for this article can be found online at: https://www.frontiersin.org/articles/10.3389/fmicb. 2021.720967/full\#supplementary-material

Supplementary Figure 1 | Verification of transcriptome data with qRT-PCR.

Supplementary Figure 2 | Bases content along raw reads.

Supplementary Figure 3 | Mean errors distribution along raw reads.

Supplementary Figure 4 | Correlation of transcriptional data.

Supplementary Table 1 | The sequences of four shRNAs for tonB gene.

Supplementary Table 2 | The sequence of Primers for PCR and qRT-PCR.

Edrees, A., Abdelhamed, H., Nho, S. W., Park, S. B., Karsi, A., Austin, F. W., et al. (2018). Construction and evaluation of type III secretion system mutants of the catfish pathogen Edwardsiella piscicida. J. Fish Dis. 41, 805-816. doi: $10.1111 /$ jfd.12784

Gomez-Santos, N., Glatter, T., Koebnik, R., Swiatek-Polatynska, M. A., and Sogaard-Andersen, L. (2019). A TonB-dependent transporter is required for secretion of protease PopC across the bacterial outer membrane. Nat. Commun. 10:1360.

Guo, L., Huang, L., Su, Y., Qin, Y., Zhao, L., and Yan, Q. (2018). secA, secD, secF, yajC, and yidC contribute to the adhesion regulation of Vibrio alginolyticus. Microbiologyopen 7:e00551. doi: 10.1002/mbo3.551

Huang, B. X., Ru, K., Yuan, Z., Whitchurch, C. B., and Mattick, J. S. (2004). tonB3 is required for normal twitching motility and extracellular assembly of type IV pili. J. Bacteriol. 186, 4387-4389. doi: 10.1128/jb.186.13.4387-4389.2004

Huang, L., Zhao, L., Qi, W., Xu, X., Zhang, J., Zhang, J., et al. (2020). Temperature-specific expression of cspAl contributes to activation of sigX during pathogenesis and intracellular survival in Pseudomonas plecoglossicida. Aquaculture 518:734861. doi: 10.1016/j.aquaculture.2019.734861

Huang, L., Zuo, Y., Jiang, Q., Su, Y., Qin, Y., Xu, X., et al. (2019). A metabolomic investigation into the temperature-dependent virulence of Pseudomonas plecoglossicida from large yellow croaker (Pseudosciaena crocea). J. Fish Dis. 42, 431-446. doi: 10.1111/jfd.12957

Huang, W., and Wilks, A. (2017). Extracellular heme uptake and the challenge of bacterial cell membranes. Annu. Rev. Biochem. 86, 799-823. doi: 10.1146/ annurev-biochem-060815-014214

Kopp, D. R., and Postle, K. (2020). The intrinsically disordered region of ExbD is required for signal transduction. J. Bacteriol. 202:e00687-19.

Li, C., Jiang, J., Xie, J., Yang, W., and Wang, Y. (2021). Transcriptome profiling and differential expression analysis of the immune-related genes during the acute phase of infection with Mycobacterium marinum in the goldfish (Carassius auratus L.). Aquaculture 533:736198. doi: 10.1016/j.aquaculture.2020.736198 
Liao, H., Liu, M., and Cheng, A. (2015). Structural features and functional mechanism of TonB in some Gram-negative bacteria-A review. Wei Sheng $W u$ Xue Bao 55, 529-536.

Liu, Z., Zhao, L., Huang, L., Qin, Y., Zhang, J., Zhang, J., et al. (2020). Integration of RNA-seq and RNAi provides a novel insight into the immune responses of Epinephelus coioides to the impB gene of Pseudomonas plecoglossicida. Fish Shellfish Immunol. 105, 135-143. doi: 10.1016/j.fsi.2020.06.023

Luo, G., Huang, L., Su, Y., Qin, Y., Xu, X., Zhao, L., et al. (2016). flrA, flrB, and $f \operatorname{lrC}$ regulate adhesion by controlling the expression of critical virulence genes in Vibrio alginolyticus. Emerg. Microbes and Infect. 5, e85. doi: 10.1038/emi.2016. 82

Luo, G., Sun, Y., Huang, L., Su, Y., Zhao, L., Qin, Y., et al. (2020). Timeresolved dual RNA-seq of tissue uncovers Pseudomonas plecoglossicida key virulence genes in host-pathogen interaction with Epinephelus coioides. Environ. Microbiol. 22, 677-693. doi: 10.1111/1462-2920.14884

Luo, G., Zhao, L., Xu, X., Qin, Y., Huang, L., Su, Y., et al. (2019). Integrated dual RNA-seq and dual iTRAQ of infected tissue reveals the functions of a diguanylate cyclase gene of Pseudomonas plecoglossicida in host-pathogen interactions with Epinephelus coioides. Fish Shellf. Immunol. 95, 481-490. doi: 10.1016/j.fsi.2019.11.008

Lupu, F., Keshari, R. S., Lambris, J. D., and Coggeshall, K. M. (2014). Crosstalk between the coagulation and complement systems in sepsis. Thromb. Res. 133, S28-S31.

Mao, L., Qin, Y., Kang, J., Wu, B., Huang, L., Wang, S., et al. (2020). Role of LuxR-type regulators in fish pathogenic Aeromonas hydrophila. J. Fish Dis. 43, 215-225. doi: 10.1111/jfd.13114

Oeemig, J. S., Ollila, O. H. S., and Iwai, H. (2018). NMR structure of the C-terminal domain of TonB protein from Pseudomonas aeruginosa. PeerJ 6:e5412. doi: $10.7717 /$ peerj. 5412

Okuda, S., Yamada, T., Hamajima, M., Itoh, M., Katayama, T., Bork, P., et al. (2008). KEGG atlas mapping for global analysis of metabolic pathways. Nucleic Acids Res. 36, W423-W426.

Park, H.-L., and Jae-Hwan, N. (2011). Regulation of innate immunity via MHC class II-mediated signaling; non-classical role of $\mathrm{MHC}$ class II in innate immunity. J. Bacteriol. Virol. 41, 205-207. doi: 10.4167/jbv.2011.41.3.205

Pontrelli, P., Grandaliano, G., and Van Kooten, C. (2020). Editorial: kidney transplantation and innate immunity. Front. Immunol. 11:603982. doi: 10.3389/ fimmu.2020.603982

Samantarrai, D., Sagar, A. L., Gudla, R., and Siddavattam, D. (2020). TonBdependent transporters in sphingomonads: unraveling their distribution and function in environmental adaptation. Microorganisms 8:359. doi: 10.3390/ microorganisms 8030359

Schauer, K., Rodionov, D. A., and de Reuse, H. (2008). New substrates for TonBdependent transport: do we only see the 'tip of the iceberg'? Trends Biochem. Sci. 33, 330-338. doi: 10.1016/j.tibs.2008.04.012

Singhapol, C., and Tinrat, S. (2020). Virulence genes analysis of Vibrio parahaemolyticus and anti-vibrio activity of the citrus extracts. Curr. Microbiol. 77, 1390-1398. doi: 10.1007/s00284-020-01941-4

Song, X., Hu, X., Sun, B., Bo, Y., Wu, K., Xiao, L., et al. (2017). A transcriptome analysis focusing on inflammation-related genes of grass carp intestines following infection with Aeromonas hydrophila. Sci. Rep. 7:40777.

Sun, Y., Zhuang, Z., Wang, X., Huang, H., Fu, Q., and Yan, Q. (2019). Dual RNAseq reveals the effect of the flgM gene of Pseudomonas plecoglossicida on the immune response of Epinephelus coioides. Fish Shellfish Immunol. 87, 515-523. doi: 10.1016/j.fsi.2019.01.041

Tang, Y., Sun, Y., Zhao, L., Xu, X., Huang, L., Qin, Y., et al. (2019). Mechanistic insight into the roles of Pseudomonas plecoglossicida clp V gene in host-pathogen interactions with Larimichthys crocea by dual RNA-seq. Fish Shellf. Immunol. 93, 344-353. doi: 10.1016/j.fsi.2019.07.066

Unanue, E. R. (2002). Perspective on antigen processing and presentation. Immunol. Rev. 185, 86-102. doi: 10.1034/j.1600-065x.2002.18510.x

Wang, L., Sun, Y., Zhao, L., Xu, X., Huang, L., Qin, Y., et al. (2019). Dual RNA-seq uncovers the immune response of Larimichthys crocea to the $\sec Y$ gene of Pseudomonas plecoglossicida from the perspective of host-pathogen interactions. Fish Shellf. Immunol. 93, 949-957. doi: 10.1016/j.fsi.2019. 08.040
Wang, Q., Liu, Q., Cao, X., Yang, M., and Zhang, Y. (2008). Characterization of two TonB systems in marine fish pathogen Vibrio alginolyticus: their roles in iron utilization and virulence. Arch. Microbiol. 190, 595-603. doi: 10.1007/s00203008-0407-1

Wieczorek, M., Abualrous, E. T., Sticht, J., Alvaro-Benito, M., Stolzenberg, S., Noe, F., et al. (2017). Major histocompatibility complex (MHC) class I and MHC class II proteins: conformational plasticity in antigen presentation. Front. Immunol. 8:292. doi: 10.3389/fimmu.2017.00292

Wiegner, R., Chakraborty, S., and Huber-Lang, M. (2016). Complementcoagulation crosstalk on cellular and artificial surfaces. Immunobiology 221, 1073-1079. doi: 10.1016/j.imbio.2016.06.005

Wilson, A. B. (2017). MHC and adaptive immunity in teleost fishes. Immunogenetics 69, 521-528. doi: 10.1007/s00251-017-1009-3

Wu, C.-C., and Chen, B.-S. (2016). A systems biology approach to the coordination of defensive and offensive molecular mechanisms in the innate and adaptive host-pathogen interaction networks. PLoS One 11:e0149303. doi: 10.1371/ journal.pone.0149303

Xin, G., Wang, F., Zhao, L., Qin, Y., Huang, L., and Yan, Q. (2020). Integration of RNA-seq and RNAi provides a novel insight into the effect of pvdE gene to the pathogenic of Pseudomonas plecoglossicida and on the immune responses of orange-spotted grouper (Epinephelus coioides). Aquaculture 529:735695. doi: 10.1016/j.aquaculture.2020.735695

Ye, H., Xu, Z., Tao, Z., Li, W., Li, Y., Yang, A., et al. (2021). Efficacy and safety of Pseudomonas plecoglossicida mutant Delta tssD-1 as a live attenuated vaccine for the large yellow croaker (Larimichthys crocea). Aquaculture 531, $1-7$.

Yu, G., Wang, L.-G., Han, Y., and He, Q.-Y. (2012). clusterProfiler: an R package for comparing biological themes among gene clusters. OMICS 16, 284-287. doi: 10.1089/omi.2011.0118

Zhang, B., Luo, G., Zhao, L., Huang, L., Qin, Y., Su, Y., et al. (2018). Integration of RNAi and RNA-seq uncovers the immune responses of Epinephelus coioides to L321_RS19110 gene of Pseudomonas plecoglossicida. Fish Shellfish Immunol. 81, 121-129. doi: 10.1016/j.fsi.2018.06.051

Zhang, M., Kang, J., Wu, B., Qin, Y., Huang, L., Zhao, L., et al. (2020). Comparative transcriptome and phenotype analysis revealed the role and mechanism of ompR in the virulence of fish pathogenic Aeromonas hydrophila. Microbiologyopen 9:e1041.

Zhang, M., Qin, Y., Huang, L., Yan, Q., Mao, L., Xu, X., et al. (2019). The role of sodA and sodB in Aeromonas hydrophila resisting oxidative damage to survive in fish macrophages and escape for further infection. Fish Shellfish Immunol. 88, 489-495. doi: 10.1016/j.fsi.2019.03.021

Zuo, Y., Zhao, L., Xu, X., Zhang, J., Zhang, J., Yan, Q., et al. (2019). Mechanisms underlying the virulence regulation of new Vibrio alginolyticus ncRNA Vvrr1 with a comparative proteomic analysis. Emerg. Microbes Infect. 8, 1604-1618. doi: $10.1080 / 22221751.2019 .1687261$

Conflict of Interest: JZ and QY were employed by company Fujian Tianma Technology Company Limited.

The remaining authors declare that the research was conducted in the absence of any commercial or financial relationships that could be construed as a potential conflict of interest.

Publisher's Note: All claims expressed in this article are solely those of the authors and do not necessarily represent those of their affiliated organizations, or those of the publisher, the editors and the reviewers. Any product that may be evaluated in this article, or claim that may be made by its manufacturer, is not guaranteed or endorsed by the publisher.

Copyright (c) $2021 \mathrm{Hu}$, Zhao, Zhuang, Wang, Fu, Huang, Lin, Huang, Qin, Zhang and Yan. This is an open-access article distributed under the terms of the Creative Commons Attribution License (CC BY). The use, distribution or reproduction in other forums is permitted, provided the original author(s) and the copyright owner(s) are credited and that the original publication in this journal is cited, in accordance with accepted academic practice. No use, distribution or reproduction is permitted which does not comply with these terms. 